\title{
Trabajo Social en el programa "Mis mejores años" del Ministerio de Inclusión Económica y Social (MIES)
}

Social work in the "my best years" program of the Ministry of Economic and Social Inclusion (MIES)

Leila María Álava Barreiro'

Ginger Katiuska Intriago Velásquez²

RECIBIDO: 20 DE ABRIL DE 2020

ACEPTADO: 5 DE JUNIO DE 2020

\section{RESUMEN}

En el marco de los cambios globales, el Ecuador a través de su Constitución del 2008, reconoce a las personas adultas mayores como grupo de atención prioritaria, lo cual implica garantizar sus derechos mediante una atención especializada tanto en el ámbito público y privado, por lo que el objetivo de esta investigación consistió en caracterizar el rol del trabajador social en el Programa "mis mejores años" del MIES en la parroquia San Sebastián del cantón Pichincha, provincia de Manabí. La

\footnotetext{
1 Magister en Educación y Desarrollo Social, Licenciada en Trabajo Social, Docente, Universidad Técnica de Manabí, Ecuador; lalava@utm.edu.ec, ORCID: https://orcid.org/0000-0001-9494-3402 Google Scholar: https://scholar.google.es/citations?hl=es\&user=DBOnsSwAAAAJ

2 Egresada de Trabajo Social, Universidad Técnica de Manabí, Ecuador; gintriago1968@utm.edu.ec, ORCID: https://orcid.org/0000-0003-0327-6558

Google Scholar: https://scholar.google.es/citations?hl=es\&user=sonznSYAAAAJ
}

Socialium revista científica de Ciencias Sociales, Vol 4 - No. 2, julio - diciembre 2020, pág. 171-180.

DOI https://doi.org/10.26490/uncp.sl.2020.4.2.560 


\section{2}

población y muestra objeto de estudio fue 75 adultos mayores beneficiarios del Programa "mis mejores años". Se fundamentó en una investigación de tipo no experimental, se utilizó el método descriptivo-exploratorio, bibliográfico, de igual manera se aplicó la técnica de la entrevista dirigida a el trabajador social responsable de ejecutar este programa en la parroquia. Como resultado se destaca la labor esencial del profesional en la aplicación de instrumentos que permiten una detección temprana de problemas en el adulto mayor, y a modo de conclusión, la intervención del profesional permite al adulto mayor involucrase en una participación activa, creando un ambiente de respeto y de restauración de derechos, reduciendo las barreras de aislamiento, fomentando la recreación en este grupo de atención prioritaria.

Palabras clave: mis mejores años, adultos mayores, inclusión económica y social

\section{ABSTRACT}

In the framework of global changes, Ecuador, through its 2008 Constitution, recognizes older adults as a group of priority care, which implies guaranteeing their rights through specialized care in both the public and private spheres, which is why The objective of this research was to characterize the role of the social worker in the MIES "My Best Years" Program in the San Sebastián parish of the Pichincha canton, Manabí province. In which we worked with 75 elderly beneficiaries of the "My best years" Program. The realization of this study was based on non-experimental research, the descriptive-exploratory, bibliographic and statistical methods were used, which allowed the information obtained to be processed, in the same way the interview technique applied to the worker was applied. responsible for executing this program in the parish. As results, the essential work of the professional in the application of instruments that allow early detection of problems in the elderly is highlighted. To conclude this program, they allow the elderly to improve their vulnerability, reducing the stereotypes that society imposes on people over 65, expanding the coverage of access to relevant services for this population group.

Keywords: my best years, older adults, economic and social inclusion 


\section{Introducción}

El Trabajador Social es un profesional que propone cambios y favorece dando soluciones a los conflictos de la sociedad, aplicando su capacidad crítica, no solamente a nivel operativo, sino que también reflexiona, piensa, analiza, investiga, descifra la realidad y contribuye al mejoramiento de las condiciones de vida de las personas quienes están carentes de recursos en situación de vulnerabilidad entre ellas las personas adultas mayores.

En esta investigación se utiliza el término "adulto mayor" por considerarlo apropiado ya que el término "viejo" está en proceso de resignificación y actualmente para gran parte de nuestra sociedad esta palabra tiene implicaciones peyorativas y se asocian a valoraciones negativas que se relacionan con términos como: Caduco, inútil, obsoleto entre otros.

Según el Consejo de igualdad e integridad (2019) afirma que "Las personas adultas mayores son las ciudadanas y ciudadanos que tienen 65 años de edad o más". Es decir que, a partir de este rango de edad, se le puede denominar de tal manera, previo al envejecimiento y la prolongación de la vida que cada individuo y toda la sociedad visualicen como una nueva forma de existencia, ya que deberán incluirse nuevos ciclos vitales que anteriormente se ignoraban.

De acuerdo a la Comisión Económica para América Latina y el Caribe (CEPAL, 2011) menciona que "existen diversos paradigmas para comprender el envejecimiento, ya que este proceso alude a una realidad multifacética atravesada no solo por el paso del calendario, sino también por aspectos fisiológicos, sociales y culturales". Definir el envejecimiento como un avance de edad es redundar en un individuo quien posee experiencias, sabidurías lo cual aprendió con el pasar de los años, sin embargo, hablar en términos específicos de los adultos mayores es agruparlos en diferentes categorías para acercarse a una comprensión definida.

Se ha clasificado a los adultos mayores en tres grupos: el viejo -joven, aquel que se encuentre entre 64 y 74 años y su funcionamiento es activo y enérgico, mientras que el viejo-viejo se encuentra entre los 75 y 84 años y con posibilidad de ser más frágil que los del grupo anterior. Y por último el viejo de edad avanzada, cuya edad es mayor a los 85 años quien con seguridad es enfermo y presenta dificultades para desarrollar sus actividades diarias. (Moreno, 2011, pp. 6-7) 


\section{4}

Continuando con este análisis, existen diferentes aspectos para hacer referencia a un adulto mayor, sin embargo, lo común entre ellos es que esta definición se acerca a partir de los 65 años y no antes de ello, es decir que el envejecimiento es algo natural en las personas, sin embargo esto implica de alguna manera dificultades en el ritmo que lleva una persona en su diario vivir, tal como lo afirma (Briones, 2018) "el envejecimiento poblacional es uno de los fenómenos de mayor impacto de nuestra época lo que ocasiona un aumento de personas de 65 años y más incrementando los índices de enfermedades crónicos- degenerativas e incapacidades".

De acuerdo al análisis del autor, el envejecimiento incrementa los indicies de enfermedades, lo que implica la salud de los adultos mayores, quienes deben estar como prioridad ante las políticas del estado, en este contexto se ejecuta el programa mis mejores años el cual busca mejorar la condición de vida de este grupo de atención prioritaria quienes se encuentren en situación de pobreza mediante la garantía de protección social o asistencia social contributiva que incluye trasferencia monetaria. en virtud de aquello, el objetivo de esta investigación es caracterizar el rol del trabajador social en el Programa "mis mejores años" del MIES en la parroquia San Sebastián del cantón Pichincha, provincia de Manabí.

\section{Desarrollo}

Mis Mejores Años se la define como una propuesta operativa del estado ecuatoriano que en base al enfoque del envejecimiento activo y saludable, busca mejorar la calidad de vida de las personas mayores de 65 años, particularmente de aquellas que se encuentran en mayores condiciones de vulnerabilidad. Con estos elementos, este programa, persigue incrementar el bienestar de las personas adultas mayores desde una mirada holística, que reconoce los aspectos individuales y sociales de las personas.

El envejecimiento desde una conceptualización natural, representa el avance prolongando de los años que vive una persona, es una etapa de regocijo y experiencias sobre diferentes aspectos esenciales de la vida, de acuerdo a la Organización Mundial de la Salud (OMS, 2020) afirma que:

La población mundial está envejeciendo a pasos acelerados, entre 2000 y 2050, la proporción de los habitantes del planeta mayores de 60 años se duplicará, pasando 
del $11 \%$ al 22\%. En números absolutos, este grupo de edad pasará de 605 millones a 2000 millones en el transcurso de medio siglo.

En ecuador, el Plan Toda una Vida favorece en la ejecución de la política pública cuyo objetivo consiste en profundizar la construcción de una sociedad incluyente, equitativa y humana; que presenta acciones orientadas a aquellos grupos que se encuentran en condiciones de pobreza y vulnerabilidad, encaminando su accionar a garantizar el ejercicio de los derechos de las personas a lo largo de su vida.

Por consiguiente, el programa mis mejores años, busca extinguir la concepción tradicional de la vejez, construida en base a un imaginario colectivo que denota una etapa caracterizada por carencias de todo nivel (económicas, físicas y sociales), enmarcando su accionar en el enfoque de los derechos lo cual conlleva un cambio paradigmático en donde se involucran aspectos en la calidad de vida permitiendo alcanzar un equilibrio y bienestar social, así lo refiere (Reyes, 2013) como "conjunto de factores que participan en la calidad de la vida de la persona y que hacen que su existencia posea todos aquellos elementos que den lugar a la tranquilidad y satisfacción humana". La calidad de vida en las personas adultas mayores requiere de diferentes elementos importantes para alcanzar un equilibrio vital y ayudar a reducir los efectos que son propios de la edad que cada persona atrae con el pasar de los años ya sean fisiológicos y patológicos.

Se precisa mencionar una diferencia entre los términos de envejecimiento fisiológico y el patológico, el primero corresponde a la forma natural con la que envejecen los órganos y diferentes partes del cuerpo, mientas que el segundo es una manera acelerada de hacerlo, y no precisamente por los años, esto se debe a factores secundarios como enfermedades o medio ambiente.

Considerando que la salud no es solo la ausencia de enfermedad, sino que involucra aspectos físico, psíquico y social. Acompañados de satisfacer las necesidades vitales como autonomía, solidaridad felicidad, entre otras, para alcanzar esto, es necesario la implementación de programas que se orienten al objetivo de mejorar el entorno de interacción de los adultos mayores que promuevan un desarrollo de actividades con sistemas de apoyo, promoción y derechos que permitan la integración e iteración en los espacios destinados para tal efecto. Entre estos, se mencionan: 


\section{6}

Centros gerontológicos residenciales: estos consisten en brindar servicios de acogida para la atención y cuidado del adulto mayor de entre 65 años o más, aquí se considera si requieren la protección de forma temporal o definitiva, se considera a quienes no pueden ser atendidos por familiares, es decir que carezcan de un lugar donde residir o se encuentren en situación de abandono y pobreza extrema en donde el alto riesgo sea eminente. También se encuentran los centros gerontológicos de atención diurna, aquí los adultos mayores asisten como complemento a su vida social, familiar y comunitaria, pues se enfoca en el mantenimiento de las facultades físicas y mentales, con ello se evita el deterioro mediante la participación con actividades acorde a su edad.

Dentro de este contexto se encuentran también los espacios activos para el envejecimiento digno, revitalización, recreación, socialización y encuentro, para ello la (Secretaría Tecnica del Plan Toda una Vida, 2018) lo define como "espacios concebidos para el encuentro, participación y socialización de personas adultas mayores sanas y aquellos que puedan trasladarse por sus propios medios físicos", es decir que éstos realizan diferentes actividades enfocadas en la motricidad también recreativas y de estimulación cognitiva, pero en algunos casos hay limitaciones de realizar estas acciones con aquellos adultos que no pueden movilizarse y que requieran una atención en el domicilio.

Continuando con estos espacios destinados para los adultos mayores, también se encuentra la atención domiciliaria, esta beneficia a los adultos cuya condición no les permite movilizarse hasta el centro a recibir la atención y dependen de otras personas para dicho efecto, en esta se incluyen actividades recreativas y del cuidado, buscando fomentar la autonomía por medio de la promoción de convivencia, participación y la relación con el medio social.

\section{Método}

El desarrollo de esta investigación se fundamentó en un tipo de estudio no experimental. Se utilizo el método descriptivo - exploratorio y bibliográfico los que permitieron ordenar la información obtenida. De la misma manera se aplicó la técnica de la entrevista y como instrumento la guía de entrevista dirigida a el trabajador social del Programa "Mis mejores años" del MIES de la parroquia San 
Sebastián del cantón Pichincha, provincia de Manabí, Ecuador. En la cual se dispuso de una población y muestra de 75 adultos mayores pertenecientes al programa.

\section{Resultados}

Se complementa la revisión bibliográfica realizada en esta investigación con la información obtenida a través de la entrevista realizada al trabajador social del programa "mis mejores años" de la Parroquia San Sebastián, éste manifiesta que dentro de las actividades realizadas están las lúdicas, las cuales buscan el desarrollo motriz con los adultos mayores, ésta favorecer el trabajo psico-motriz y cognitivo de los participantes, también se fomenta el autoestima, el respeto y la buena convivencia entre todos, de igual manera se fomenta un cuidado preventivo, progresivo e integral en los adultos mayores con ejercicios de expresión corporal. En este sentido el profesional realiza gestiones con entidades gubernamentales como el Gobierno autónomo descentralizado Parroquial, y el Municipal, coordinando el proceso de intervención en la cual realiza conferencias, aplicación de instrumentos, acompañamiento en casos ocasionales que involucren al adulto mayor.

En este contexto se realizan las visitas domiciliarias, en la cual se aplican fichas de acuerdo a las condiciones de cada adulto mayor, entre las más aplicadas están la ficha de admisión e ingreso, esta consiste informar al adulto sobre el programa y en ella asume su integración al mismo, también se detecta la condición física, psicología y el estado de salud, de igual manera la convivencia del usuario. Continuando con los instrumentos de aplicación se encuentra la ficha de valoración social, la cual consiste en obtener información referente a la vivienda y redes de apoyo disponibles en el adulto mayor, también se indaga en el estado de salud, nutrición y situación económica. De igual manera se aplica la ficha mini examen del estado mental, esta involucra acciones para detectar la condición del estado en la memoria de los usuarios con actividades y ejercicios prácticos que permiten detectar alguna irregularidad en ellos, ésta se aplica cada tres meses.

La ejecución de las visitas domiciliarias involucran una preparación previa aplicando acciones de logística y planificación en la cual se coordina con el MIES para la capacitación que se requiere con los adultos mayores, estas se enfocan en sexualidad, vulnerabilidad, derechos, cuidado personal, respeto al prójimo entre 


\section{8}

otras, integrando acciones que se realizaran como los cambios de horarios, las gestiones con el personal del Ministerio de Salud, para la revisión cotidiana, también se coordina con la policía nacional quienes colaboran en la seguridad del personal. La función del profesional en trabajo social es amplia, sin embargo, esta no se limita a una característica en concreta, sino más bien va encaminada a coordinar acciones con distintas instituciones que velan por dar mejoras a las personas adultas mayores, tal como lo es con fundaciones, centros de salud, instituciones educativas, centros de salud, y entidades no gubernamentales. En este sentido, el Trabajador social es gestor, asesor, coordinador, facilitador y capacitador en los distintos procesos que lleva a cabo el programa mis mejores años.

\section{Discusión}

El programa mis mejores años está orientado a fortalecer un sistema de inclusión y equidad social, permitiendo la acción del Trabajo Social en una perspectiva incluyente ejecutando un sistema de cuidados que garantice la protección y atención integral y especial para la población adulta mayor de 65 años. Tal como lo señala la (Comunidad Valenciana , 2018), quien menciona "el aumento de personas mayores o ancianas es enorme, así como las demandas de atención y cuidados que precisan. Es por esto que la figura del trabajador social es fundamental y cada vez adquiere más importancia". Sin embargo, para llevar a cabo un adecuado abordaje, se requiere de la disponibilidad de recursos y la participación de las personas involucradas. Pero este profesional debe estar con plenos conocimientos de la realidad y de su entorno. así lo expresa (Guerrini, 2010) "El profesional que aspira servir de ayuda a otros debe tener un conocimiento de sí mismo, de sus fortalezas y debilidades personales que puedan impedir el trabajo efectivo con otros". Todo esto como base la preparación académico-científica que se imparte desde las universidades formadoras de conocimientos.

Con respecto al proceso de abordaje social que se realiza en Trabajo Social del programa Mis Mejores Años, este aplica instrumentos para realizar una valoración en distintos ámbitos del usuario, así lo señala La Organización Panamericana de la Salud (2016), "La evaluación del funcionamiento social puede servir para determinar los factores protectores con los que cuenta el individuo para su bienestar y los factores 
de riesgo que necesitan vigilancia y quizás intervención psicosocial". Es indispensable considerar cada aspecto como importante en el adulto mayor, sin omisión de ningún tipo de situación, se deben identificar lo económico, afectivo como relación directa con el bienestar así lo señala (Moya, 2016) quien afirma:

Debemos atender también que al valorar el entorno socio-familiar es fundamental la identificación y evaluación del cuidador principal en sus posibilidades de continencia completa o parcial, considerando que la capacidad de contener a otro implica tres aspectos esenciales: económico, afectivo, instrumental.

El cuidador principal es imprescindible para el correcto desempeño del adulto mayor, siendo una importante conexión de apoyo para el efectivo goce del bienestar emocional y social del usuario, quien requiere la atención pertinente

\section{Conclusión}

El trabajador social perteneciente al programa mis mejores años del Ministerio de Inclusión Económica y Social, es un profesional que tiene la capacidad y el conocimiento acorde a las exigencias sociales, impulsa procesos de inclusión al grupo de atención prioritaria, ejecuta acciones, coordina con instituciones para alcanzar el objetivo en el usuario. De igual manera es un evaluador en el proceso de intervención para alcanzar el bienestar físico psicológico y social en las personas que interviene.

Las actividades que ejecuta el profesional en trabajo social, permiten al adulto mayor involucrase en una participación activa, creando un ambiente de respeto y de restauración de derechos, reduciendo las barreras de aislamiento, fomentando la recreación en este grupo de atención prioritaria, aplicando de manera adecuada las políticas del estado en programas que reducen significativamente estos índices. Finalmente, el programa mis mejores años, permiten al adulto mayor mejorar su condición de vulnerabilidad, reduciendo los estereotipos que la sociedad impone a las personas mayores de 65 años, ampliado la función del profesional a cargo de dicho programa, permitiéndole asumir roles de facilitador, capacitador, gestor, educador, coordinador, lo que implica la aplicación del conocimiento tórico metodológico de su proceso formativo para desempeñar adecuadamente estas acciones. 


\section{0}

\section{Referencias}

Briones, D. (2018). Derecho Ecuador. Obtenido de https://www.derechoecuador.com/ley-del-adulto-mayor

CEPAL. (Junio de 2011). Comisión Económica para América Latina y el Caribe. Obtenido de http://www.cepal.org/celade/envejecimiento

Comunidad Valenciana . (2018). Lares. Obtenido de https://www.larescvalenciana.org/trabajador-social-importancia-vidaanciano/

Guerrini, M. (2010). La Vejez, su abordaje desde el Trabajo Social. Margen057, 10. Integeneracional, C. N. (2019). Consejo Nacional para la Igualdad Integeneracional . Obtenido de https://www.igualdad.gob.ec/personas-adultas-mayoressituacion-y-derechos/

Moreno, J. P. (201 1). Manual de practica basica del adulto mayor. En F. L. Morales Aguilar, Practica Basica del Adulto Mayor (págs. 6-7). Mexico: El Manual Moderno.

Moya, J. I. (12 de Enero de 2016). Revista Medica Electronica. Obtenido de Portales Médicos: https://www.revista-portalesmedicos.com/revista-medica/elpaciente-geriatrico/

OMS. (2020). Organizacion Mundial de la Salud. Obtenido de https://www.who.int/ageing/about/facts/es/

Organizacion Panamericana de la Salud. (14 de Julio de 2016). Informed. Obtenido de Red de Salud de Cuba: http://www.sld.cu/galerias/pdf/sitios/gericuba/modulo7.pdf

Reyes, O. (2013). Teoría del bienestar y el óptimo de pareto como problemas microeconómicos. Revista Electrónica de Investigación en Ciencias Económicas Abriendo Camino al Conocimiento, 221.

Secretaría Tecnica del Plan Toda una Vida. (2018). Toda una Vida. Obtenido de https://www.todaunavida.gob.ec/wpcontent/uploads/2018/09/Documento-proyecto-Plan-Toda-una-Vida.pdf 